\title{
Erratum to: IGF-II inhibitory DNAzymes inhibit the invasion and migration of hepatocarcinoma cells
}

Min Zhang $\cdot$ Hang Zhao $\cdot$ Fangyue Luo $\cdot$

Su Luo - Wei Shi

Published online: 19 March 2011

(C) Springer Science+Business Media B.V. 2011

\section{Erratum to: Biotechnol Lett \\ DOI 10.1007/s10529-011-0529-4}

Unfortunately, first and fourth author's family name and given names had been wrongly published.
The correct version is Min Zhang, not Zhang Min and $\mathrm{Su}$ Luo not Luo Su as published.

The online version of the original article can be found under doi:10.1007/s10529-011-0529-4.

M. Zhang $\cdot$ H. Zhao $\cdot$ W. Shi $(\bowtie)$

Key Laboratory for Molecular Enzymology and

Engineering, The Ministry of Education, Jilin University,

Room 226, Life Science Building, Qianjin Street 2699,

Changchun 130012, China

e-mail: shiw@jlu.edu.cn

M. Zhang $\cdot$ F. Luo $\cdot$ S. Luo

Beihua University, Huashan Road 3999,

Jilin 132100, China 